\title{
Correlación entre la microestructura y el comportamiento plástico a alta temperatura de compuestos TZP-Ni (40\% vol.)
}

\author{
A. MORALES-RODRÍGUEZ, A. BRAVO-LEÓN, A. DOMÍNGUEZ-RODRÍGUEZ Y M. JIMÉNEZ-MELENDO \\ Departamento de Física de la Materia Condensada, Universidad de Sevilla, Apto. 1065, 41080 Sevilla, España
}

\begin{abstract}
Se ha estudiado la microestructura y el comportamiento mecánico a alta temperatura de compuestos de circona tetragonal (3Y-TZP) con un $40 \%$ en volumen de níquel (referidos en adelante como TZP-Ni). El contenido en níquel está por encima del punto de percolación, que en estos compuestos cerámico-metal procesados en vía húmeda se sitúa en el 34\% vol. de contenido metálico. La caracterización microestructural se ha realizado mediante observaciones de microscopía electrónica de barrido. La plasticidad de los compuestos se ha estudiado mediante ensayos de fluencia en compresión uniaxial a carga constante en atmósfera de argón realizados en el rango de temperaturas entre 900 y $1250{ }^{\circ} \mathrm{C}$ y tensiones entre 9 y $144 \mathrm{MPa}$. Para estudiar el efecto del níquel en el comportamiento del cermet se ensayaron en condiciones similares esqueletos de TZP, obtenidos mediante la eliminación de la fase metálica de los compuestos por ataque químico con $\mathrm{HNO}_{3}$. El comportamiento plástico de estos materiales está controlado por la matriz cerámica. Las velocidades de deformación son muy superiores a las del TZP monolítico, hecho que se ha explicado atendiendo a la distribución de la fase metálica en la matriz en estos compuestos. La percolación de la fase metálica es responsable del aumento en la velocidad de deformación y permite que el cermet deforme a temperaturas tan bajas como $900{ }^{\circ} \mathrm{C}$.
\end{abstract}

Palabras clave: plasticidad, cermet, TZP, percolación, esqueleto.

\section{Correlation between microstructure high-temperature plastic behavior in TZP-Ni $(40 \%)$ composites}

Microstructure and high-temperature mechanical behaviour of TZP-Ni composites with 40 vol.\% of nickel above the percolation threshold (placed in $34 \mathrm{vol} \%$ in these wet-processed ceramic-metal composites) has been studied. The microstructure has been characterized using scanning electron microscopy. The composite plasticity has been studied by means of uniaxial compressive creep tests under constant load in argon atmosphere carried out in the temperature range between 900 and $1250{ }^{\circ} \mathrm{C}$ and stresses between 9 and $144 \mathrm{MPa}$. TZP skeleton has been deformed in similar conditions to study nickel effect in global cermet behavior. The metallic phase has been removed from the cermets using chemical etching with $\mathrm{HNO}_{3}$. The plastic behavior of these materials is controlled by the ceramic matrix. The deformation rates are higher than monolithic TZP ones. This fact has been explained taking into account the metallic phase distribution into the matrix in these composites. Metal percolation provides materials with high ductility allowing the cermets to deform at temperatures as low as $900{ }^{\circ} \mathrm{C}$.

Keywords: plasticity, cermet, TZP, percolation, skeleton.

\section{INTRODUCCIÓN}

Desde un punto de vista teórico, los compuestos cerámicometálicos (cermets) son materiales interesantes, entre otros motivos, por ser idóneos para contrastar las predicciones de las teorías de percolación (1), ya que sus propiedades eléctricas, por ejemplo, son muy sensibles a la interconexión de la fase metálica. En particular, los compuestos TZP-Ni estudiados son especialmente atractivos porque presentan valores extraordinariamente altos del umbral de percolación (34\% vol. de metal) en comparación con la fracción volumétrica crítica clásica correspondiente a una distribución aleatoria de la fase metálica $(16 \%)(1)$, que se ha asociado con la existencia de un cierto ordenamiento del metal en la matriz (2).

Las aplicaciones para las que se han propuesto estos materiales, como protectores térmicos en placas con función y microestructura gradual, en la fabricación de sensores de flujo y temperatura y como ánodos de células de combustible de electrolito sólido para convertir energía química en eléctrica y calorífica (3-5), requieren la evaluación de su respuesta mecánica en las condiciones de operación (sometidos a temperaturas altas en atmósferas variables) para optimizar su viabilidad. El objetivo de este trabajo es analizar el efecto de la percolación de la fase metálica en el comportamiento plástico, por lo que se han estudiado compuestos con un contenido en níquel del $40 \%$ vol., por encima del umbral de percolación (2), y se ha comparado su comportamiento con TZP monolítico y con esqueletos de circona obtenidos a partir de los cermets.

\section{PROCEDIMIENTO EXPERIMENTAL}

\subsection{Fabricación}

Los materiales utilizados en este trabajo, cuyas propiedades eléctricas han sido estudiadas en el Instituto de Ciencias de Materiales de Madrid (ICMM) (2), fueron procesados según el procedimiento descrito en (6). Los materiales se prepararon a partir de una mezcla de polvos comerciales de: (i) $\mathrm{ZrO}_{2}$ estabilizado en su fase tetragonal (TZP) con $3 \%$ molar de $\mathrm{Y}_{2} \mathrm{O}_{3}$ 
(TZ-3YS; Tosoh Corp.) de alta pureza (análisis químico en \% en peso: $\mathrm{ZrO}_{2}$ 95.000, $\mathrm{Y}_{2} \mathrm{O}_{3} 4.98, \mathrm{Al}_{2} \mathrm{O}_{3} 0.005, \mathrm{Fe}_{2} \mathrm{O}_{3} 0.004, \mathrm{Na}_{2} \mathrm{O}$ 0.003, $\mathrm{SiO}_{2}$ 0.002); y (ii) Ni ultrafino (NFP-401; Kawatetsu Mining Co., Ltd) con una pureza del $99 \%$ en peso (análisis químico en $\%$ en peso: $\mathrm{Ni}>99.9, \mathrm{Cl} 0.002, \mathrm{C} 0.04-0.06, \mathrm{Fe}$ 0.002-0.005, Co 0.002, Mn 0.001, Cr 0.001, Na 0.001, K 0.001), sin considerar el contenido en oxígeno (0.2-0.4\% en peso). Los polvos de partida fueron homogeneizados en vía húmeda mediante la preparación de suspensiones en agua destilada con una proporción de sólidos del $70 \%$ en peso a las que se añadió un 3\% de defloculante (Dolapix PC-33); la proporción relativa de níquel fue del $40 \%$ en volumen para la obtención del cermet. Tras una molienda con bolas de circona durante 24 $\mathrm{h}$, las suspensiones se secaron en una estufa a $90^{\circ} \mathrm{C}$. Los polvos resultantes se compactaron mediante prensado isostático en frío a $207 \mathrm{MPa}$ en forma de bloques prismáticos de $4 \times 4 \times 40$ $\mathrm{mm}^{3}$ y se sinterizaron posteriormente en atmósfera reductora $90 \% \mathrm{Ar} / 10 \% \mathrm{H}_{2}$ a $1430{ }^{\circ} \mathrm{C}$, por debajo de la temperatura de fusión del níquel $\left(1455{ }^{\circ} \mathrm{C}\right)$, durante $2 \mathrm{~h}$. Durante el calentamiento $\left(10^{\circ} \mathrm{C} / \mathrm{min}\right)$, antes de alcanzar la temperatura de sinterización, se mantuvo el material a $500{ }^{\circ} \mathrm{C}$ durante $2 \mathrm{~h}$ para reducir el $\mathrm{NiO}$ presente en las partículas iniciales a $\mathrm{Ni}$ metálico. El mismo procedimiento, pero sin adición de níquel, se siguió para obtener TZP monolítico.

A partir de muestras cortadas de los compuestos recibidos, se obtuvieron estructuras de esqueleto de TZP. El níquel se eliminó mediante ataque químico con $\mathrm{HNO}_{3}$ a temperatura ambiente durante $100 \mathrm{~h}$ aproximadamente. La eliminación total de la fase metálica fue controlada mediante el pesado de las muestras.

\subsection{Caracterización de los materiales}

La densidad de los materiales se determinó mediante la medida de sus dimensiones con un tornillo micrométrico y su pesado con una balanza de precisión (la densidad teórica del cermet se calculó empleando la regla de las mezclas, $\left.\rho_{\mathrm{c}}=\mathrm{f}_{\mathrm{TZP}} \rho_{\mathrm{TZP}}+\mathrm{f}_{\mathrm{Ni}} \rho_{\mathrm{Ni}}\right)$. La densidad de las estructuras esqueleto se deduce de forma inmediata contabilizando como poros el volumen de níquel eliminado, $\mathrm{f}_{\mathrm{Ni}}$.

La caracterización microestructural de los materiales se realizó mediante microscopía electrónica de barrido (MEB) (Servicio de Microscopía Electrónica, Universidad de Sevilla). Los estudios se han realizado en un microscopio Philips XL-30 equipado con un detector de electrones retrodispersados que permite el contraste de composición en las imágenes.

Para las observaciones de MEB, se cortaron secciones de las muestras mediante una sierra de borde adiamantado de $0.15 \mathrm{~mm}$ de grosor y se pulieron con pasta de diamante de hasta $1 \mu \mathrm{m}$. Los granos de níquel se han estudiado mediante la observación de las superficies pulidas. Para el estudio de la matriz de circona, sin embargo, las superficies pulidas fueron atacadas térmicamente durante $30 \mathrm{~min}$. a $1250{ }^{\circ} \mathrm{C}$ en atmósfera de $90 \% \mathrm{Ar} / 10 \% \mathrm{H}_{2}$ para revelar las fronteras de grano. Finalmente, sobre las superficies de observación se evaporó oro para evitar la acumulación de carga. En el caso de las muestras deformadas, se observaron secciones paralelas al eje de aplicación de la carga. Mediante un analizador de imágenes semiautomático se midió el tamaño de grano, $\mathrm{d}$,

definido como el diámetro plano equivalente $\mathrm{d}=\left(\frac{4 \cdot \text { área }}{\pi}\right)^{1 / 2}$ , el factor de forma, $F=4 \pi \frac{\text { área }}{{\text { (perímetro })^{2}}^{2}}$ y el ángulo de orientación, $\theta$, definido como el ángulo que forma el diámetro máximo del grano respecto de una dirección prefijada (en el caso de las muestras deformadas, la dirección de aplicación de la carga).

\subsection{Ensayos mecánicos a alta temperatura}

Se han realizado ensayos de fluencia a carga constante en atmósfera de argón para evitar la oxidación de las partículas metálicas, en un rango de temperaturas entre 900 y $1250{ }^{\circ} \mathrm{C}$ y a tensiones entre 9 y $144 \mathrm{MPa}$ utilizando una máquina de ensayos prototipo (7). Los ensayos mecánicos se realizaron sobre muestras de dimensiones aproximadas $3 \times 3 \times 4 \mathrm{~mm}^{3}$ cortadas a partir de los materiales recibidos con el uso de una sierra de precisión y pulidas mecánicamente con un disco abrasivo de $30 \mu \mathrm{m}$ para eliminar los defectos del corte y asegurar que las muestras tenían forma de prisma recto de sección cuadrada. Los datos registrados: variación de la altura de la muestra en función del tiempo se representaron en curvas $\log \dot{\varepsilon}-\varepsilon$ (curvas de fluencia), donde $\dot{\varepsilon}$ y $\varepsilon$ son la velocidad de deformación y la deformación, respectivamente. El análisis de las curvas se ha realizado admitiendo que la deformación plástica se rige por la ecuación clásica de fluencia:

$$
\dot{\varepsilon}=A \sigma^{\mathrm{n}} \mathrm{d}^{-\mathrm{p}} \exp (-\mathrm{Q} / \mathrm{RT}),
$$

donde A es una constante, $\sigma$ la tensión, $\mathrm{n}$ el exponente de tensión, d el tamaño de grano, p el exponente de tamaño de grano, $Q$ la energía de activación, $\mathrm{R}$ la constante universal de los gases y $\mathrm{T}$ la temperatura absoluta. Mediante la variación de las condiciones de solicitación durante las experiencias (saltos de carga o temperatura) se determinaron los parámetros de fluencia ( $\mathrm{n}$ ó $\mathrm{Q}$, respectivamente), que informan sobre los mecanismos de deformación.

\section{RESULTADOS}

\subsection{Caracterización de los materiales estudiados}

Las densidades relativas medidas fueron del $90 \%$ para el material TZP monolítico y del $85 \%$ para el cermet TZP-Ni. La disminución de la densidad relativa del compuesto respecto al TZP monolítico se atribuye al empeoramiento de los proceso de densificación de la matriz de TZP por la adición de níquel

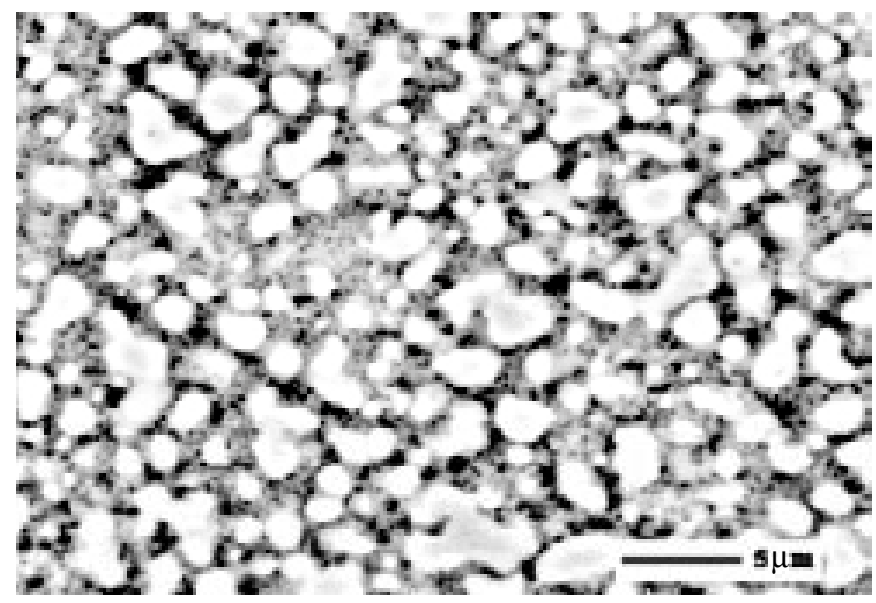

Fig. 1- Micrografía MEB a bajos aumentos del compuesto TZP-Ni ( $40 \%$ vol.). La porosidad (negro) se encuentra asociada, generalmente, a las inclusiones metálicas (blanco). 

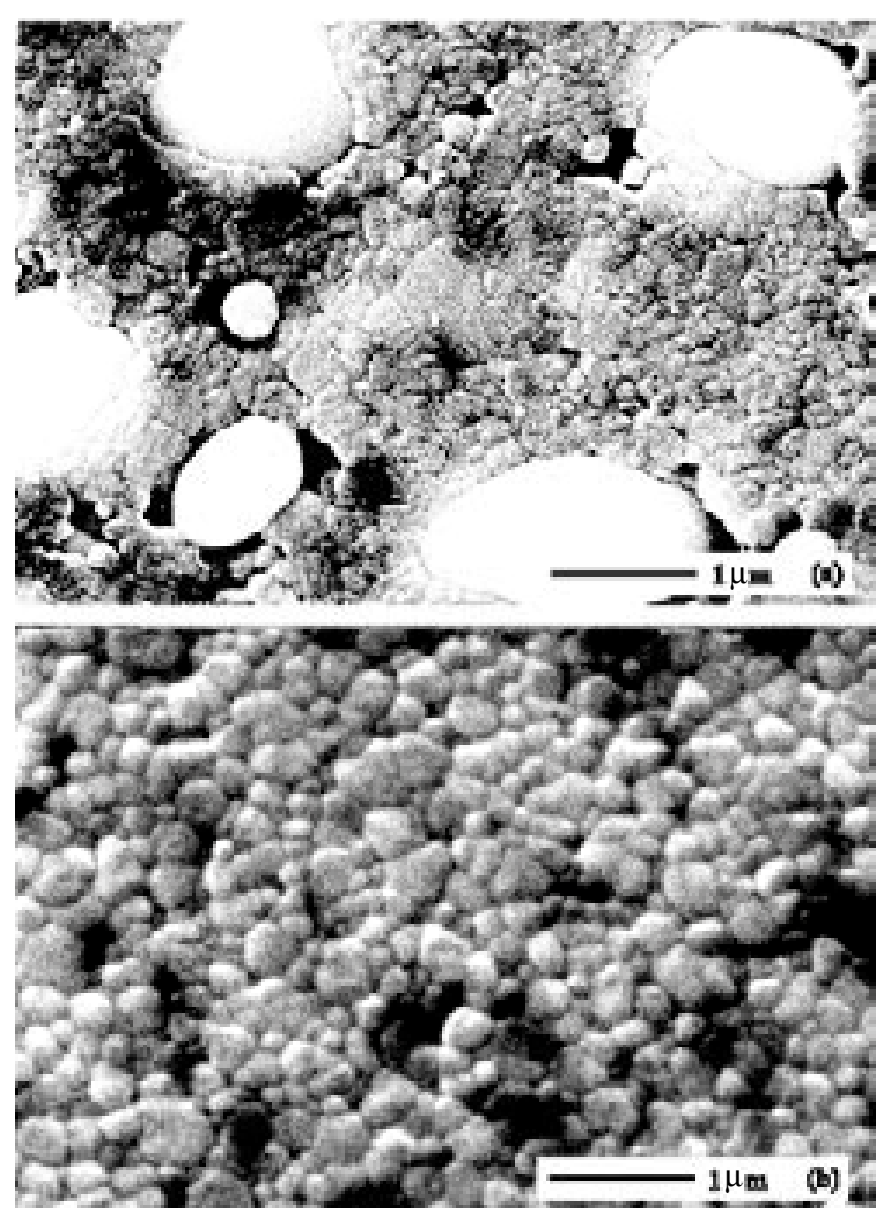

Fig. 2- Microestructura de la circona de los materiales estudiados: (a) compuesto TZP-Ni (40\% vol.); (b) TZP monolítico.

(2). Los esqueletos de circona tienen una densidad del $46 \%$, estimándose un 5\% de níquel residual máximo.

Las observaciones mediante MEB de los materiales compuestos corroboraron la alta porosidad de los mismos (los poros se distinguen por su color negro (figura 1)). En la micrografía se observa también un contacto apreciable entre las inclusiones metálicas (fase clara), de acuerdo con el hecho de que el punto de percolación esté situado en el $34 \%$ de contenido en níquel (2). El tamaño medio de los granos metálicos es de aproximadamente $1.2 \mu \mathrm{m}$ y con un factor de forma $\mathrm{F} \approx 0.8$, sin presentar orientaciones preferentes dado que las muestras han sido sinterizadas sin carga. La matriz de circona (fase oscura) presenta granos equiaxiados más finos que los de níquel con un tamaño de grano $(\mathrm{d} \approx 0.13$ $\mu \mathrm{m}$ ) (figura 2a) ligeramente menor que el de la circona en el material monolítico $(\mathrm{d} \approx 0.22 \mu \mathrm{m})$ (figura $2 \mathrm{~b}$ ). En todos los casos, se trata de granos equiaxiados $(\mathrm{F} \approx 0.9)$ y sin orientación privilegiada. La figura 3 muestra la estructura del esqueleto de circona (fase clara) con los huecos dejados por el níquel que se ha retirado (fase oscura).

\subsection{Curvas de fluencia}

\subsubsection{Comportamiento plástico de TZP-Ni}

La figura 4 muestra las curvas de fluencia para el compuesto TZP-Ni (símbolos rellenos) y su esqueleto (símbolos huecos) ensayados a tensiones entre 9.5 y $14 \mathrm{MPa}$ en el rango
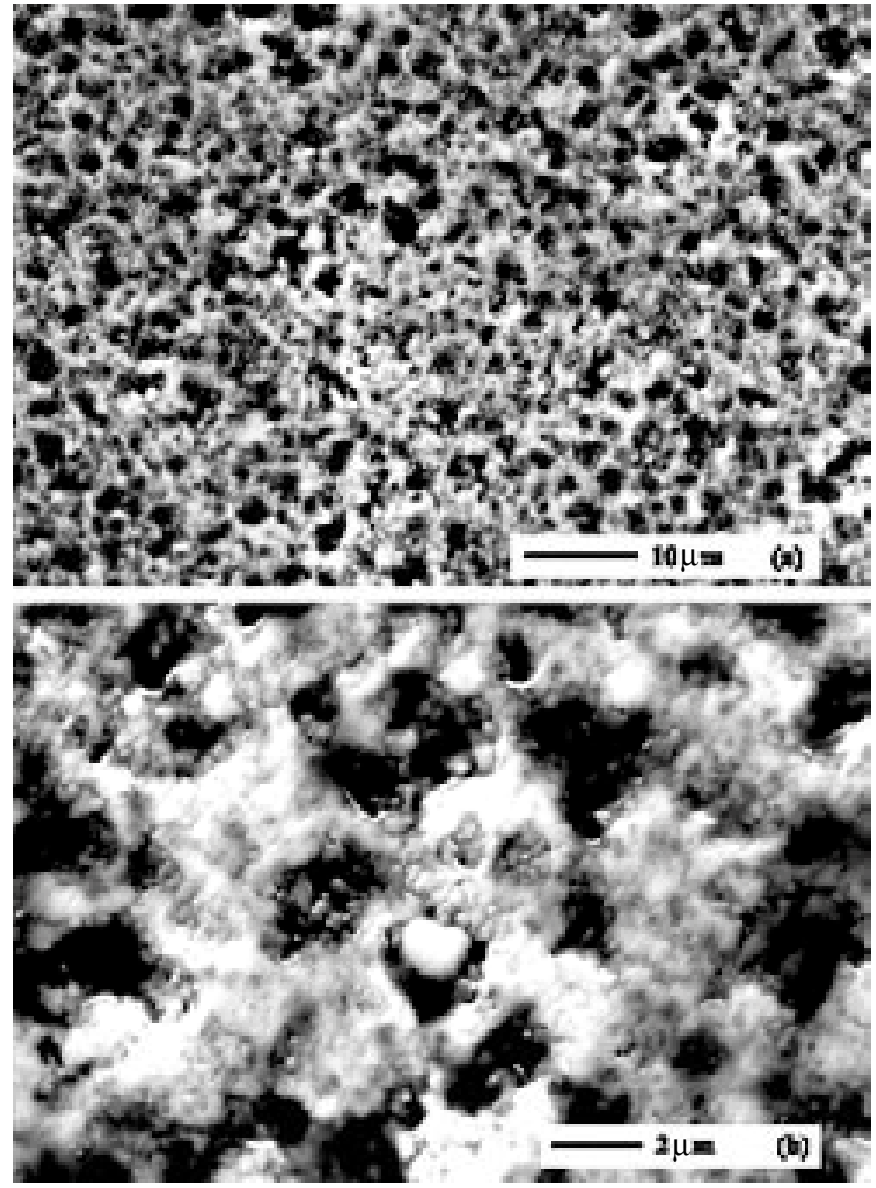

Fig. 3- Micrografías MEB del esqueleto de circona del compuesto: (a) general; (b) detalle de la distribución de los huecos dejados por el níquel.

de temperaturas entre 1200 y $1250{ }^{\circ} \mathrm{C}$. Se observa que la deformación alcanza rápidamente un estado estacionario tras la aplicación de las diferentes cargas y los saltos de temperatura. Se encuentra que los comportamientos plásticos del cermet y de su esqueleto son idénticos, presentando los tramos de deformación superpuestos. Cabe destacar, por tanto, que la ausencia de fase metálica no modifica ni las velocidades de deformación ni los parámetros de fluencia. Los valores de $\mathrm{n}$ y $\mathrm{Q}$ medidos en los cambios de $\sigma$ y $\mathrm{T}$ se indican en la figura, obteniéndose un promedio de $\mathrm{n}=3.9 \pm 0.1$ y $\mathrm{Q}=640 \pm 40$ $\mathrm{kJ} / \mathrm{mol}$ en los dos casos. Aunque ambos materiales alcanzaron deformaciones grandes $(\varepsilon \approx 50 \%)$, el esqueleto presentaba daños macroscópicos tras los ensayos, como pérdida de los vértices, lo que sugiere una menor ductilidad.

El elevado ritmo de deformación y la gran ductilidad observados en el material TZP-Ni (figura 4) han permitido bajar la temperatura de deformación manteniendo valores de la velocidad de deformación apreciables. La figura 5 muestra las curvas de fluencia a tensiones entre 60 y $72 \mathrm{MPa}$ entre 1050 y $1000{ }^{\circ} \mathrm{C}$. En estas condiciones, se midieron valores de $\mathrm{n}$ menores, $\mathrm{n}=2.6 \pm 0.1$, mientras que no se apreciaron cambios en $\mathrm{Q}$. Al igual que antes, se encuentran comportamientos similares en el cermet y el esqueleto, salvo que hay signos de daño en el último tras la deformación.

Esta reducción de la ductilidad se pone de manifiesto de modo más evidente en condiciones de ensayo más severas (figura 6), temperatura muy baja (entre 950 y $900{ }^{\circ} \mathrm{C}$ ) y tensiones altas ( $\geq 100 \mathrm{MPa}$ ). En este ensayo se han obtenido 


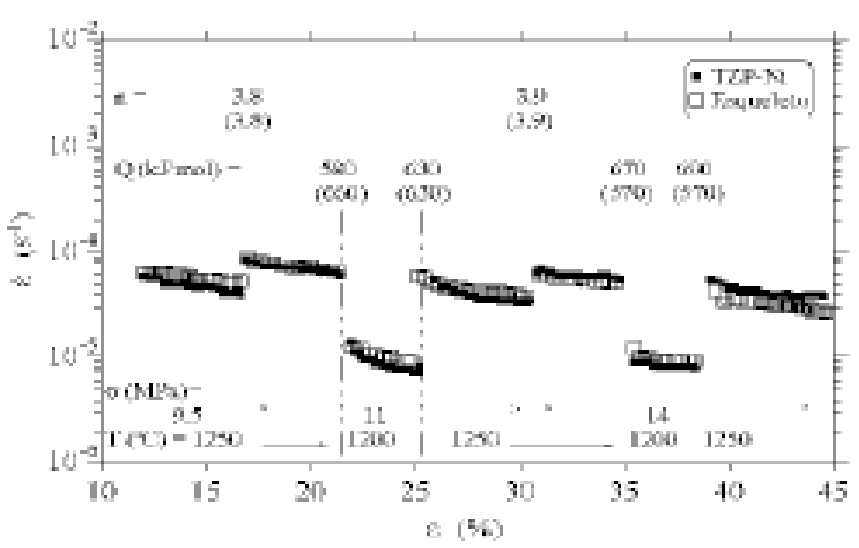

Fig. 4- Comparación de las curvas de fluencia del compuesto con el esqueleto a tensiones entre 9.5 y $14 \mathrm{MPa}$ y temperaturas entre 1250 y $1200{ }^{\circ} \mathrm{C}$. Se muestran los valores del exponente de tensión n y de la energía de activación $\mathrm{Q}$ para el compuesto (y esqueleto).

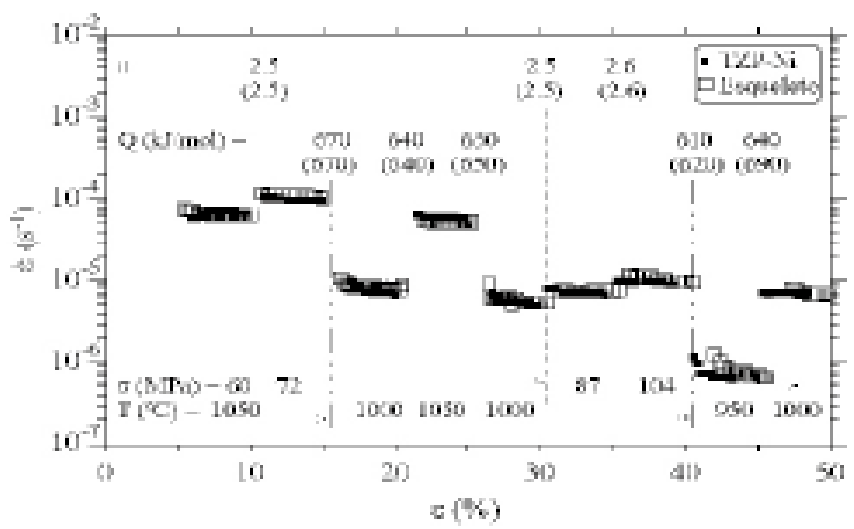

Fig. 5- Comparación de las curvas de fluencia del compuesto con el esqueleto a tensiones entre 60 y $104 \mathrm{MPa}$ y temperaturas entre 1050 y $1000^{\circ} \mathrm{C}$.

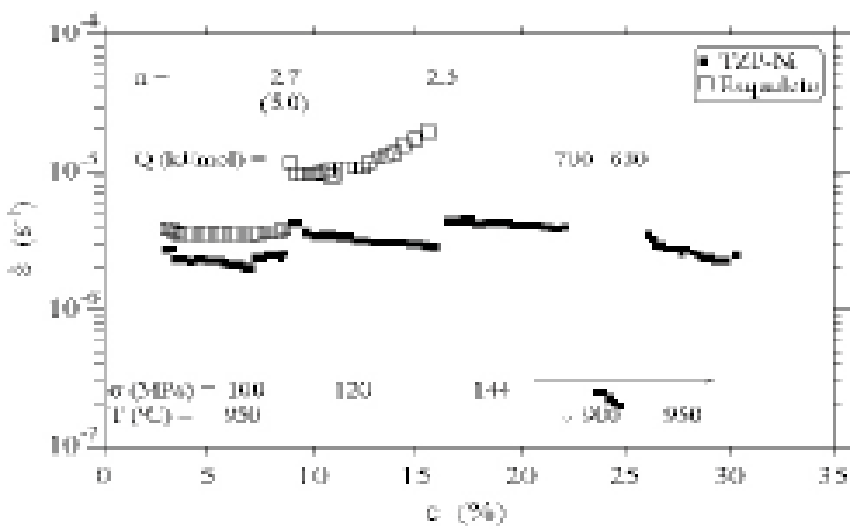

Fig. 6- Comparación de las curvas de fluencia del compuesto con el esqueleto a $950{ }^{\circ} \mathrm{C}$.

valores de $\mathrm{n}=2.6 \pm 0.1$ y de $\mathrm{Q}=670 \pm 40 \mathrm{~kJ} / \mathrm{mol}$ similares a los anteriores (figura 5). Hay que destacar que el esqueleto se fractura para $\varepsilon \approx 15 \%$ en contraste con el comportamiento del cermet, que alcanzó una deformación del 30\% sin signos macroscópicos de daño (8). También hay que señalar que para temperaturas por debajo de $1000{ }^{\circ} \mathrm{C}$ no existen resultados previos sobre la fluencia de compuestos a base de circona.

\subsubsection{Comportamiento plástico del TZP monolítico}

Con el fin de poder establecer comparaciones, se estudió el comportamiento de la circona monolítica procesada por el mismo método que los compuestos de níquel (6). Sin embargo, dado el mayor tamaño de grano y la mayor resistencia a la deformación de este material, dicho estudio no se pudo realizar en las mismas condiciones experimentales.

La figura 7 muestra la curva de fluencia del TZP entre 1250 y $1300{ }^{\circ} \mathrm{C}$ y en un rango de tensiones entre 11 y $16 \mathrm{MPa}$. Se obtuvo un exponente de tensión $\mathrm{n}=2.9 \pm 0.1$ y una energía de activación de $Q=680 \pm 20 \mathrm{~kJ} / \mathrm{mol}$. Estos parámetros, junto con los valores de $\varepsilon$ medidos, permiten asegurar que se trata de un material de alta pureza -de acuerdo con el contenido de impurezas tan bajo de los polvos de partida-, del que ya se ha estudiado su comportamiento mecánico en amplias revisiones $(9,10)$.

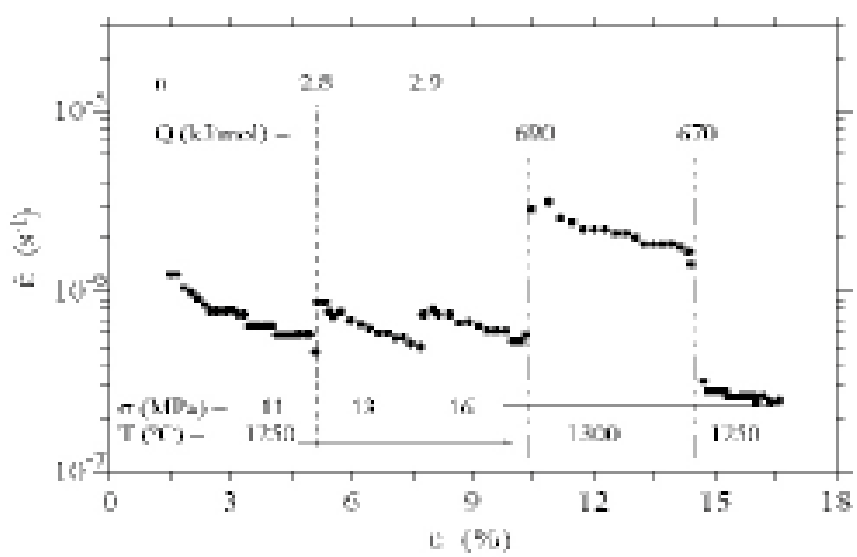

Fig. 7- Curva de fluencia de TZP monolítico.

\subsection{Caracterización de los materiales deformados}

Independientemente de las condiciones de ensayo, se ha encontrado sistemáticamente, que las inclusiones metálicas (fase clara, figura 8) cambian de forma durante el proceso

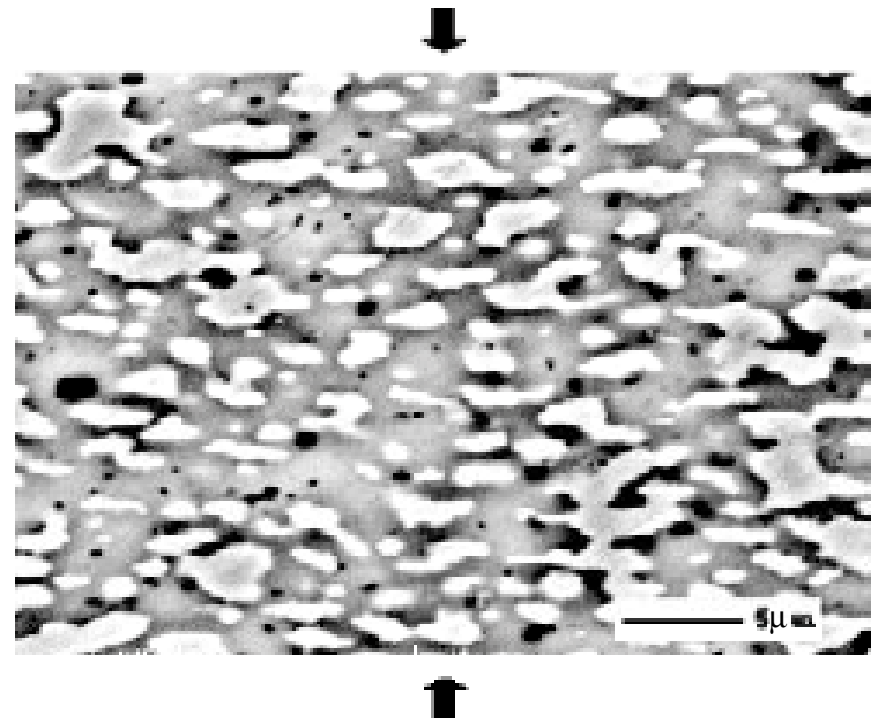

Fig. 8-Micrografía MEB a bajos aumentos del compuesto deformado ( 9.5-14 MPa; $\left.1250-120{ }^{\circ} \mathrm{C}\right)$. El eje de compresión se indica con flechas. 


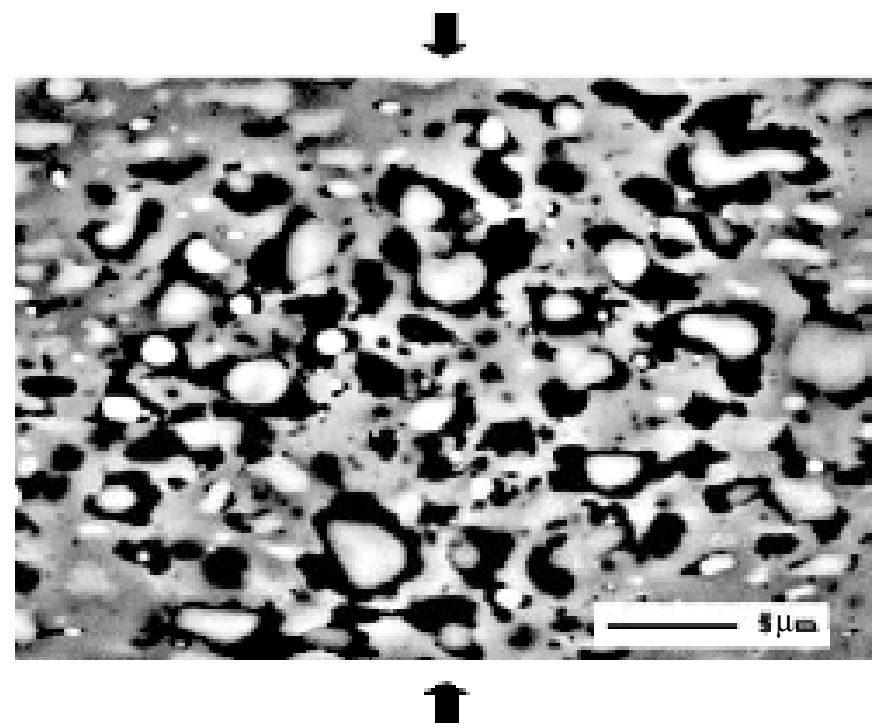

Fig. 9- Zona con defecto de níquel en el compuesto deformado.

de deformación plástica, adquiriendo un aspecto alargado $(\mathrm{F} \approx 0.6)$ orientándose preferentemente con el eje mayor perpendicular al eje de aplicación de la carga. El tamaño de grano medio de las inclusiones es ligeramente mayor $(\mathrm{d}=1.3$ $\mu \mathrm{m})$ y la distribución de tamaños es notoriamente más ancha que antes de la deformación, poniendo de manifiesto que la deformación favorece el contacto entre los granos, ya que no es posible resolver los granos individuales.

A partir de las micrografías ha sido posible estimar que las densidades relativas de las muestras deformadas es inferior al $95 \%$ en todos los casos. En el caso del TZP monolítico, la densidad relativa es del 100\% tras la deformación.

No se han producido variaciones significativas de la microestructura de la matriz durante la deformación, en buen acuerdo con la ausencia de crecimiento de grano a temperaturas por debajo de $1250^{\circ} \mathrm{C}$ en TZP monolítico de alta pureza (11).

En los compuestos TZP-Ni deformados se ha observado una pérdida de homogeneidad en la distribución del níquel en la matriz. La figura 9 muestra una región donde hay ausencia de inclusiones de níquel. Estas zonas defectuosas son muy numerosas, se encuentran repartidas de forma homogénea y son de un tamaño regular, de aproximadamente unas $20 \mu \mathrm{m}$. Se han descrito microestructuras similares en compuestos SiCSi que presentaban regiones libres de Si tras la deformación (12).

\section{DISCUSIÓN}

\subsection{Análisis de los resultados experimentales}

Como se indicó anteriormente, los datos obtenidos en este trabajo para el TZP monolítico se sitúan en los valores correspondientes a la circona de alta pureza. Respecto al compuesto TZP-Ni, los valores de $\mathrm{n}$ y $\mathrm{Q}$, así como su evolución con la temperatura y la tensión son muy similares a los del TZP monolítico puro (10), sugiriendo este hecho que su comportamiento está controlado fundamentalmente por la fase cerámica. El hecho de que los esqueletos de circona presenten los mismos valores de los parámetros de fluencia y de la velocidad de deformación que los cermets apoya que el control de la plasticidad se deba a la fase dura, como se ha atribuido también en otros cermets (12-14).

Admitiendo que la circona controla la velocidad de deformación de los compuestos y por comparación con los resultados del TZP monolítico, se puede corregir el efecto del tamaño de grano usando un exponente del tamaño de grano $\mathrm{p}=2$ (10). La figura 10 muestra la variación de la velocidad de deformación (corregida con el tamaño de grano), $\dot{\varepsilon} \cdot \mathrm{d}^{2}$, con la tensión, $\sigma$, para TZP de alta y baja pureza (de (10)), junto con los datos de este trabajo. Se encuentra una diferencia considerable en el valor de la velocidad de deformación del compuesto comparado con el TZP, en un factor de 60, mientras que el TZP estudiado coincide, efectivamente, con los datos existentes en la literatura para circonas puras.

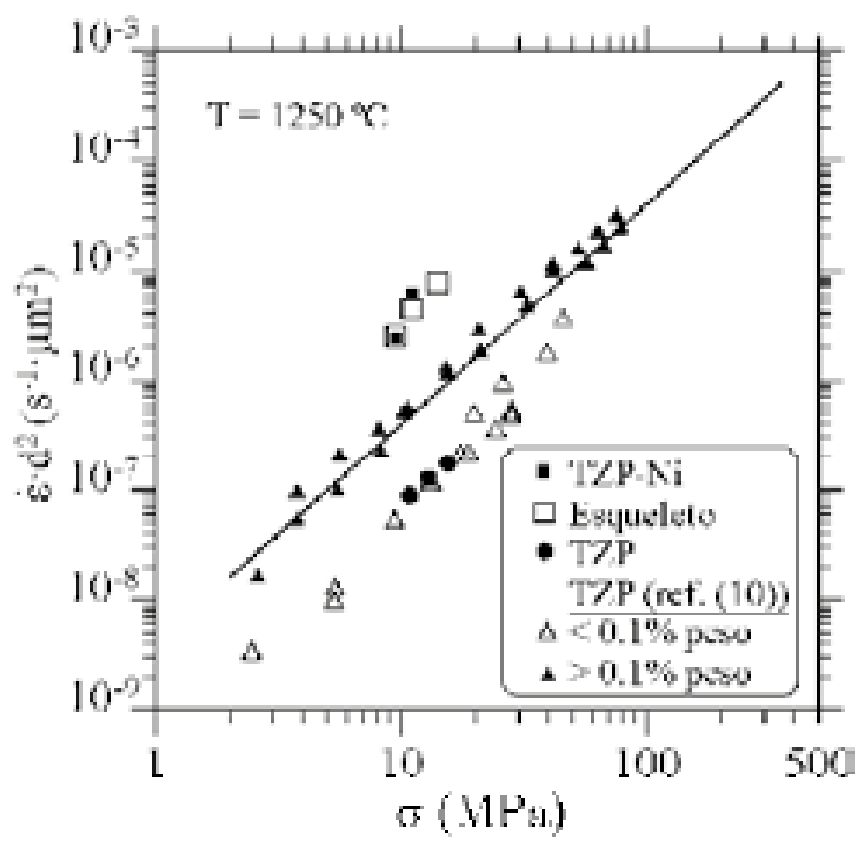

Fig. 10- Velocidad de deformación (normalizada con el tamaño de grano) frente a la tensión a $1250{ }^{\circ} \mathrm{C}$ para TZP de alta y baja pureza (10), incluyendo los datos de este trabajo.

\subsection{Efecto del níquel percolado en el comportamiento plástico}

Las velocidades de deformación del compuesto TZP-Ni (40\% vol.) pueden explicarse adecuadamente teniendo en cuenta que: (i) el níquel no ofrece resistencia a la deformación en comparación con la circona (15); y (ii) la particular distribución de la fase metálica en el compuesto. El níquel, al encontrarse interconectado, puede fluir libremente en el material desde las zonas en compresión hacia aquéllas en tracción como sugieren las regiones libres de níquel (figura 9). Admitiendo que el níquel no ofrece ninguna resistencia a la deformación (como se deduce de que las velocidades de deformación de los esqueletos son idénticas a las de los cermets), la fase metálica interconectada equivale a efectos de soporte de carga a un aumento de la porosidad real del material, haciendo posible tratar el compuesto como una circona porosa. 
Se han desarrollado modelos de mínima área sólida (MSA del inglés Minimum Solid Area) para explicar la dependencia con la porosidad de la resistencia a la deformación plástica (16). Según estos modelos, propuestos por R. W. Rice, las estructuras porosas se pueden modelar mediante apilamientos regulares de partículas huecas entre las que se ubica la estructura sólida, siendo posible el cálculo de las áreas sólidas resultantes de los posibles cortes transversales de estas estructuras. El comportamiento mecánico depende de las zonas que soportan la máxima tensión, es decir, las de área mínima perpendicular a la tensión aplicada.

Considerando que el níquel percolado se comporta como los poros de aire a efectos del soporte de carga, como se indicó anteriormente, se pueden modelar los materiales idealizando los poros y las inclusiones metálicas del cermet (o los huecos dejados por las mismas en las estructuras esqueleto) como un apilamiento cúbico de poros esféricos $(16,17)$. La tensión efectiva que soporta el material, $\sigma_{\text {ef }}$, y la tensión aplicada, $\sigma$, están relacionadas con la porosidad del material según la expresión:

$$
\sigma_{\mathrm{ef}}=\frac{\sigma}{1-\frac{\pi}{4}\left(\frac{6 \mathrm{P}}{\pi}\right)^{2 / 3}},
$$

de modo que la tensión responsable de la deformación de los compuestos TZP-Ni (40\%) es muy superior a la tensión aplicada, ya que hay que incluir la fracción volumétrica de níquel como porosidad del compuesto, resultando una porosidad efectiva $\mathrm{P}=0.49$.

La figura 11 presenta los valores de $\dot{\varepsilon} \cdot \mathrm{d}^{2}$, frente a la tensión efectiva, $\sigma_{\text {ef }}$, calculada mediante la ecuación (2) para el cermet (y el esqueleto) a $1250{ }^{\circ} \mathrm{C}$, junto con los datos del TZP. Como puede observarse, una vez corregida la tensión, los materiales estudiados presentan prácticamente las mismas velocidades de deformación que el TZP monolítico. Es importante señalar que este resultado confirma que la presencia de porosidad y la percolación de la fase metálica son responsables de los valores tan elevados de las velocidades de deformación en estos compuestos.

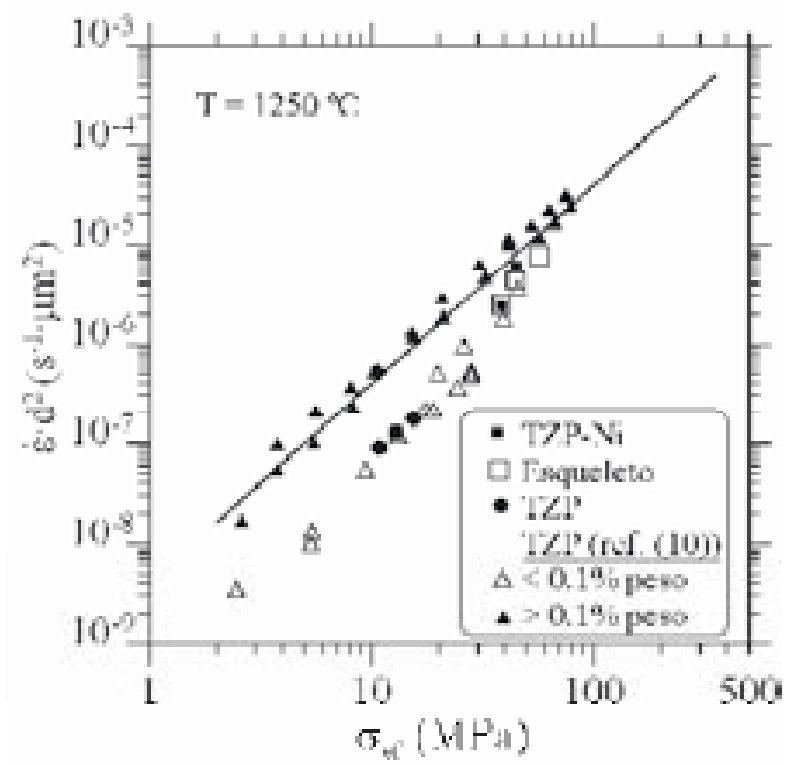

Fig. 11- Velocidad de deformación (normalizada con el tamaño de grano) frente a la tensión efectiva (corregida del efecto de la porosidad) a $1250{ }^{\circ} \mathrm{C}$ para los materiales estudiados.

\section{CONCLUSIONES}

Se ha estudiado la microestructura y el comportamiento plástico a alta temperatura de compuestos TZP-Ni $(40 \%$ vol.) con contenido de fase metálica por encima del punto de percolación (que en este sistema se sitúa en el 34\%) con las siguientes conclusiones:

(i) Los compuestos recibidos tienen una densidad relativa del $85 \%$. La microestructura consiste en dos fases continuas interconectadas de TZP y Ni. Los granos de ambas fases son equiaxiados. El tamaño medio de los granos de níquel $(1.2 \mu \mathrm{m})$ es diez veces superior al tamaño de los granos de la matriz $(0.13 \mu \mathrm{m})$.

(ii) Los cermets fueron deformados en compresión entre 900 y $1250{ }^{\circ} \mathrm{C}$ a tensiones entre 9 y $144 \mathrm{MPa}$, en atmósfera de argón. Los parámetros de fluencia presentan el mismo comportamiento que en la circona monolítica de alta pureza, indicando que la matriz de TZP controla la deformación plástica. Los valores de la velocidad de deformación exhibida por los compuestos (aproximadamente 60 veces la del TZP monolítico) pueden explicarse mediante la porosidad y la interconexión de la fase metálica. Los esqueletos de circona presentan velocidades de deformación idénticas a las de los cermets, señalando que el níquel no soporta carga en la estructura, y en fluencia el compuesto se puede describir como una circona porosa considerando el níquel como poros.

(iii) Las inclusiones metálicas evolucionan a formas alargadas durante la deformación. La ausencia de níquel en algunas regiones indica que la deformación favorece la conexión entre granos de $\mathrm{Ni}$ y su redistribución en la estructura. Los granos de la fase cerámica permanecen inalterados.

\section{AGRADECIMIENTOS}

Este trabajo ha sido financiado mediante el proyecto CICYT $\mathrm{n}^{\circ}$. MAT2000-1117 y n ${ }^{\circ}$. MAT2003-04199-C02-02 (Ministerio de Ciencia y Tecnología, España).

\section{BIBLIOGRAFÍA}

1. D. S. McLachlan, M. Blaszkiewicz, R. E. Newnham. «Electrical Resistivity of Composites». J. Am. Ceram. Soc. 73 [7] 2187-2203 (1990)

2. C. Pecharromán, S. López-Esteban, J. F. Bartolomé, , J. S. Moya. «Evidence of Nearest-Neighbor Ordering in Wet-Processed Zirconia-Nickel Composites». J. Am. Ceram. Soc. 84 [10] 2439-2441 (2001).

3. S. Reschke, C. Bodganow. «Engineering Ceramics: New Perspectives through Value-Added (Multi-) Functionality». Key Eng. Materials 175-176, 1-10 (1999).

4. J. E. Sundeen, R. C. Buchanan. «Electrical properties of nickel-zirconia cermet films for temperature- and flow-sensor applications». Sensor. Actuators A-Phys. 63 [1] 33-40 (1997).

5. S. T. Aruna, M. Muthuraman, K. C. Patil. «Synthesis and properties of NiYSZ cermet: anode material for solid oxide fuel cells». Solid State Ionics 111, 45-51 (1998).

6. S. López-Esteban. «Procesamiento, caracterización eléctrica y mecánica de materiales compuestos 3Y-TZP/Ni». Tesis Doctoral, Universidad Politécnica de Madrid, 2001.

7. H. Gervais, B. Pellicier, J. Castaing. «Machine de fluage pour essays en compression à hautes temperatures de matériaux céramiques». Rev. Int. Htes. Temp. et Réfract. 15, 43-47 (1978).

8. A. Morales-Rodríguez, A. Bravo-León, A. Domínguez-Rodríguez, M. Jiménez-Melendo. «Creep of zirconia/nickel composites». Mater. Sci. Forum 447-448, 347-352 (2003).

9. A. H. Chokshi. «Diffusion creep in oxide ceramics». J. Eur. Ceram. Soc. 22, 2469-2478 (2002). 
10. M. Jiménez-Melendo, A. Domínguez-Rodríguez, A. Bravo-León «Superplastic Flow of Fine-Grained Yttria-Stabilized Zirconia Polycrystals: Constitutive Equation and Deformation Mechanisms». J. Am. Ceram. Soc. 81 [11] 2761-2776 (1998).

11. T.-G. Nieh, J. Wadsworth. «Dynamic Grain Growth During Superplastic Deformation of Yttria-Stabilized Zirconia Polycrystals». J. Am. Ceram. Soc. 72 [8] 1469-1472 (1989).

12. J. Martínez-Fernández, A. Muñoz, A. R. de Arellano-López, F. M. VarelaFeria, A. Domínguez-Rodríguez, M. Singh. «Microstructure-mechanical properties correlation in siliconized silicon carbide ceramics». Acta mater. 51 [11] 3259-3275 (2003).
13. A. R. de Arellano-López, K. C. Goretta, E. T. Park, S. E. Dorris, U. Balchandran, J. L. Routbort. "High-temperature deformation of a $\mathrm{BaCe}_{0.8} \mathrm{Y}_{0.2} \mathrm{O}_{3-\mathrm{y}}+\mathrm{Ni}$ composite». J. Eur. Ceram. Soc. 22, 2555-2560 (2002).

14. S. Bolognini, G. Feusier, D. Mari, T. Viatte, W. Benoit. «TiMoCN-based cermets: high-temperature deformation». Int. J. Refr. Metals Hard Mater 21, 19-29 (2003)

15. H. J. Frost, M.F. Ashby. «The F.C.C. metals: $\mathrm{Ni}, \mathrm{Cu}, \mathrm{Ag}, \mathrm{Al}, \mathrm{Pb}$ and $\square-\mathrm{Fe}$ », pp. 20-24 en Deformation Mechanism Maps: The Plasticity and Creep of Metals and Ceramics. Pergamon Press, Oxford (UK) 1982.

16. R. W. Rice. «Evaluation and extension of physical property-porosity models based on minimum solid area». J. Mater. Sci. 31, 102-118 (1996).

17. R. W. Rice, pp. 72-76 en Porosity of ceramics. Ed. M. Dekker Inc., New York (EE.UU.) 2000

Recibido: 09.01 .05

Aceptado: 26.04 .05 